Revue internationale de pédagogie de

l'enseignement supérieur

$36(2) \mid 2020$

Numéro spécial - été 2020

\title{
Accompagner à la littéracie universitaire par le dialogue
}

Claire Polo

\section{(2) OpenEdition}

Journals

Electronic version

URL: http://journals.openedition.org/ripes/2767

DOI: 10.4000/ripes. 2767

ISSN: 2076-8427

Publisher

Association internationale de pédagogie universitaire

Electronic reference

Claire Polo, "Accompagner à la littéracie universitaire par le dialogue", Revue internationale de pédagogie de l'enseignement supérieur [Online], 36(2) | 2020, Online since 23 November 2020, connection on 05

February 2021. URL: http://journals.openedition.org/ripes/2767 ; DOI: https://doi.org/10.4000/ripes. 2767

This text was automatically generated on 5 February 2021.

Article L.111-1 du Code de la propriété intellectuelle. 


\title{
Accompagner à la littéracie universitaire par le dialogue
}

\author{
Claire Polo
}

\section{Introduction}

1 Le dispositif Créer Lire Ecrire et Faire de la Science pour l'Université (CLEFS), expérimenté depuis novembre 2018 à Lyon 2, fournit un accompagnement renforcé à quelques étudiants et étudiantes de première année de licence pour prévenir leur décrochage, ou plutôt favoriser leur accrochage aux études supérieures qu'ils ont choisi d'entreprendre. Si les moyens utilisés pour sa mise en œuvre proviennent du "parcours de remédiation » prévu dans la loi $\mathrm{ORE}^{1}$, l'approche pédagogique choisie s'écarte du modèle déficitaire de réparation de lacunes supposées et prend plutôt appui sur la valorisation des ressources des personnes volontairement engagées dans le dispositif. L'ensemble du parcours s'organise autour d'un travail collaboratif en mode projet portant sur une thématique interdisciplinaire, qui est l'occasion de travailler trois blocs de compétences, relatives à la méthodologie et la littéracie universitaires, ainsi qu'au développement de l'esprit critique et scientifique. Les normes académiques correspondant à ces trois dimensions ne font pas l'objet d'un enseignement formel, mais sont appréhendées conjointement comme outils indispensables pour mener à bien le projet envisagé, dont l'aboutissement est la création d'un évènement public ${ }^{2}$. En effet, un double pari pédagogique préside à la conception de ce dispositif. D'une part, il s'agit de chercher à donner du sens à l'apprentissage de ces compétences, notamment en accordant une place centrale à l'établissement de liens entre le travail universitaire et la vie à l'extérieur de l'université et en montrant comment leur maîtrise éclaire toute tentative d'appréhension $d u$ fonctionnement du monde social. D'autre part, une spécificité fondamentale du dispositif est la prise en compte attentive des dimensions socioaffectives en jeu dans l'entrée à l'université. Dans ce contexte d'enseignement relativement exceptionnel (travail en groupes de 10 à 12 à raison de $6 \mathrm{~h}$ hebdomadaires), l'approche didactique de l'écrit qui est développée relève de l'enseignement dialogique. Une première partie de cet article présente l'ancrage 
théorique qui fonde les choix d'ingénierie pédagogique opérés. Dans un second temps, je reviens sur le contexte de travail étudié, aux plans institutionnel et pédagogique. Enfin, un dernier volet détaille le traitement didactique réservé à la lecture et à l'écriture au sein de ce dispositif.

\section{Ancrage théorique et principes guidant l'ingénierie pédagogique}

2 L'accompagnement, en tant que posture professionnelle, suppose un engagement éthique de la part des accompagnants et accompagnantes qui les amène à continuellement ajuster leur comportement entre plusieurs pôles structurant leur activité. A ce titre, Paul (2004) a souligné qu'il s'agit souvent de naviguer entre «ingénierie " (instrumentation technique planifiée) et «ingéniosité »(habileté et créativité professionnelles permettant l'adaptation adéquate à ce qui survient). Mon propos se concentre ici sur la présentation des principes, théoriquement fondés, guidant l'ingénierie pédagogique du dispositif CLEFS, à savoir l'accompagnement des étudiants et étudiantes tel qu'il a été pensé et organisé a priori, même si, bien entendu, des ajustements ont nécessairement ensuite lieu dans sa mise en œuvre.

\subsection{L'enseignement-apprentissage : un processus cognitif, relationnel et affectif}

3 Le travail d'ingénierie pédagogique qui a donné naissance au dispositif CLEFS s'appuie sur une conception de l'enseignement-apprentissage comme un processus à la fois cognitif, relationnel et affectif. Dans la lignée des recherches internationales menées dans les sciences de l'apprendre (learning sciences), il s'agit de considérer tout apprentissage comme impliquant l'engagement dans une interaction avec autrui, à propos d'un objet du monde tel qu'il est représenté dans une situation didactique, permettant de transformer, en les rendant plus performantes, les manières d'être, de faire, ou de penser à son égard. Ce processus d'avancement cognitif s'appuyant à la fois sur la confrontation à un objet de savoir (savoir-faire, savoir-être) et sur la possibilité d'une certaine intersubjectivité, il ne saurait advenir sans la médiation des émotions en jeu dans la situation didactique, tant dans le rapport au savoir qu'aux personnes impliquées. Le titre de l'ouvrage coordonné par Baker et al. (2013), résume bien cette triple nature de l'apprentissage : Affective Learning Together.

Dans une telle perspective, une place importante est donnée au conflit sociocognitif (Doise et Mugny, 1981) et aux effets affectifs qu'il suppose en termes d'alternance de phases de tension et de détente, notamment dès lors qu'on s'intéresse aux situations didactiques favorisant l'apprentissage collaboratif (Baker et al., 2009). Comme dans l'ensemble des travaux s'appuyant sur une conception socioconstructiviste de l'apprentissage, le langage, dans une telle perspective, occupe une place centrale. Ainsi, nombre de travaux étudient les formes de discours entre élèves les plus bénéfiques pour l'apprentissage de savoirs disciplinaires, et/ou les façons d'enseigner ces formes de raisonnement verbal (Mercer, 1996; Asterhan, 2013). La notion d'argumentation tient ici une place importante: au-delà du fait d'argumenter pour construire de nouvelles connaissances (arguing to learn), il s'agit également d'enseigner l'argumentation comme ensemble de compétences transversales à rechercher pour 
elles-mêmes (learning to argue) (Von Aufschnaiter et al., 2008). Si, dans ces travaux, des modalités écrites sont parfois mobilisées dans la situation didactique, les compétences de lecture et d'écriture qu'elles supposent ne sont généralement pas réellement problématisées.

5 Aborder le problème de la littéracie, et donc de la place de l'écrit dans cette dynamique socio-cognitivo-affective de l'apprentissage, oblige à porter le regard sur d'autres espaces-temps que celui du dialogue instantané, et à s'intéresser à d'autres modalités de communication. On peut se situer à peine en amont ou en aval de l'interaction didactique elle-même, si l'on s'intéresse à une lecture qui précède la discussion, ou à un travail d'écriture qui lui succède. Plus fondamentalement, l'écrit permet également de démultiplier les possibilités de conflits sociocognitifs en dialoguant en différé, ou avec une personne géographiquement éloignée. Pour la trace qu'il garde d'une pensée, l'écrit assure également une fonction de gardien mémoriel, et favorise la réflexivité, une forme de dialogue ultérieur de la personne ou du groupe avec des idées formulées au moment de l'écriture. Fondamentalement, la lecture permet d'être confronté à la pensée d'une autre personne sans être en co-présence; l'écriture de confronter la sienne à d'autres dans un autre espace-temps. Dès lors que la littéracie elle-même devient un objet d'enseignement, il s'agit de didactiser un ensemble de compétences transversales associées à la lecture et à l'écriture, dans un champ social donné.

\subsection{Sortir du modèle déficitaire : accompagner à la littéracie plutôt que remédier à l'illetrisme}

6 Le terme de littéracie, issu de recherches anglophones, est de plus en plus utilisé en France depuis les années 2000, tant dans le vocabulaire académique que politique voire médiatique. Une conception simpliste de cette notion conduit souvent à employer ce terme comme contraire de l'illettrisme, soit comme la maitrise de compétences de production et de réception de l'écrit nécessaires à l'intégration dans la société (Rispail, 2011). Par exemple, on en appelle aux mutations de la société sous l'effet du développement des nouvelles technologies de l'information et de la communication et de l'entrée dans une économie de la connaissance pour démultiplier les compétences littéraciques indispensables à tout citoyen. Ainsi, le terme littéracie s'est vu décliné en littéracie numérique, littéracie scientifique, etc. Dès lors, la tâche éducative en la matière consisterait à remédier à l'illettrisme de certaines personnes ou catégories de personnes trop éloignées de ces compétences littéraciques fondamentales. L'enjeu est de taille : on risquerait de ne pas être tout à fait apte au métier étudiant sans littéracie universitaire comme on ne serait pas tout à fait citoyen sans littéracie numérique ou scientifique. Cette tendance à didactiser la littéracie comme on combat l'illettrisme est d'autant plus plausible qu'elle conforte une représentation commune dans le champ éducatif qui consiste à n'attribuer les difficultés rencontrées par certains publics qu’à des déficiences personnelles. L'approche didactique fondée sur ce modèle déficitaire suppose d'identifier les lacunes spécifiques des élèves, puis de les combler par des interventions spécifiques (Tessier et Schmidt, 2007).

7 L'approche didactique guidant le dispositif CLEFS prend ses distances avec ce modèle déficitaire en s'appuyant sur une conception plus complexe de la littéracie. Au-delà de ce sens premier de possession de compétences fondamentales de lecture et d'écriture, elle est considérée dans une acception plus large, impliquant la familiarisation avec des 
pratiques lettrées socialement situées, ici associées à la culture universitaire. Ainsi, la perspective retenue est celle du champ d'étude sur les littéracies universitaires, entendu comme «la description des pratiques et des genres de l'écrit en contexte universitaire » (Delcambre et Lahanier-Reuter, 2010, p. 25). Dans cette optique, plutôt que de pointer des lacunes, l'enseignement doit partir des savoir-faire des personnes, pour les accompagner vers des pratiques de lecture et d'écriture spécifiques au milieu auquel ils sont en train de se socialiser, à savoir l'université. Il y a là un véritable changement de regard et de posture didactique : «il ne s'agit plus de voir les groupes sociaux dans lesquels nous intervenons [...] comme constitués de "manques", de "blancs", de "vides", à remplir ou combler coûte que coûte, mais comme des acteurs déjà intégrés dans une société et donc déjà porteurs de savoirs et de compétences » (Rispail, 2011, p. 2).

$8 \mathrm{Au}$ plan théorique, une telle approche est fondée sur une conception de la littéracie comme un continuum de compétences, dont l'apprentissage va des tout premiers contacts avec le mode écrit à l'acquisition de normes hyperspécialisées liées à un champ professionnel spécifique, et, en particulier, à l'écrit scientifique (Delcambre et Lahanier-Reuter, 2010, p. 26). Au-delà du traitement didactique spécifique de la littéracie, cette posture relève d'un changement de paradigme dans la façon d'intervenir auprès de ces publics, à savoir le passage de la remédiation des difficultés à l'accompagnement vers la réussite (Reverdy, 2017). Plutôt que de chercher à rattraper un retard défini par les normes des programmes scolaires, et de combler les lacunes des élèves ${ }^{3}$ supposant la transmission de savoirs et de compétences dans une relation d'aide asymétrique, l'accompagnement suppose notamment, via une relation moins verticale, la mise en place d'une situation didactique permettant aux personnes de progresser à leur rythme dans la construction active de leurs apprentissages.

Une telle posture rejoint les définitions de l'accompagnement, proches, proposées respectivement par Paul (2012) : «se joindre à quelqu'un pour aller où il va en même temps que lui » (p.14) et par Beauvais (2004) : «aider une personne à cheminer, à se construire, à atteindre ses buts " (p.101). Il ne s'agit dès lors pas tant de fixer un objectif de l'extérieur, soit l'acquisition des normes de l'écrit universitaire en progressant dans l'acquisition de la littéracie académique, mais de cheminer avec les personnes accompagnées dans leur rapport à la littéracie, en mobilisant leurs pratiques préexistantes de recours à la lecture et à l'écriture. En respectant leur autonomie vis-àvis des apprentissages, ici littéraciques, l'enjeu est de favoriser son développement via la compréhension du sens des normes académiques en question, afin de leur permettre, dans les termes de Holec (1991), « d'auto-diriger » leur progression en se construisant des objectifs, contenus et évaluations selon leur propre élan pour apprendre. Cependant, à la suite de Little, qui travaille, dans la lignée de Holec (1991), sur l'autonomie de l'apprenant en langues étrangères, il convient de souligner qu'une telle capacité à prendre en charge son propre apprentissage suppose un engagement volontaire et réflexif qui ne saurait se développer que si la personne s'estime en mesure de mener à bien cet auto-accomplissement (sense of selffulfillment) (Little, 2010, p. 28-29). En effet, si la rhétorique de l'autonomie dans l'apprentissage conduit à rendre quelqu'un seul responsable de sa réussite ou de son échec sans un véritable travail d'accompagnement, elle risque de ne fonctionner que comme écho culpabilisant voire psychologisant des «institutions de soi» (Ehrenberg, 2000). Selon Beauvais, une éthique de l'accompagnement soucieuse d'éviter cet écueil doit ainsi être fondée non seulement sur les principes de retenue et de doute, mais également sur un principe de 
responsabilité qui reconnaît à l'accompagnant ou l'accompagnante une «métaresponsabilité ", soit la responsabilité de soutenir la responsabilité de l'autre en l'aidant à développer une véritable autonomie (Beauvais, 2004, p. 108). Le potentiel d'une telle posture réside en effet dans l'articulation des processus post-modernes d'individualisation et d'autonomisation avec un renouvellement de la socialisation au sein de la relation d'accompagnement, ce que Paul (2004) appelle la substitution au "principe de réparation» du bénéficiaire réifié le "principe de reliance» de l'accompagné, conçu comme acteur de sa propre évolution (p. 306). Ainsi, la personne accompagnée n'est plus «objet» de politiques sociales mais sujet capable, dans la relation d'accompagnement, de définir ses besoins et de travailler à comment atteindre ses objectifs.

\subsection{L'accompagnement par le projet collaboratif}

10 Depuis quelques décennies, les dispositifs spécifiquement conçus pour prendre en charge une partie du public de l'institution scolaire, considérée comme particulièrement fragile, se multiplient (Félix et Saujat, 2008). Certains y voient une avancée permettant de donner un coup de pouce aux personnes en difficulté, d'autres l'aveu des limites de l'institution, et de son incapacité à prendre en compte la diversité des rythmes d'apprentissage des élèves via une différenciation efficace en classe (Feyfant, 2016). Dans un contexte de massification de l'entrée à l'université, on assiste à la montée d'une problématique similaire de décrochage d'une partie des élèves. Cet effet touche inégalement les différentes filières universitaires, la philosophie, l'histoire ou le droit étant par exemple moins touchées que la sociologie ou la psychologie, semblant souvent plus accessibles aux personnes issues de milieux socioéconomiques plus modestes (Soulié, 1995, Perret, 2007). L'accès à l'enseignement supérieur de nouveaux profils de bacheliers, notamment issus des lycées technologiques et professionnels, pose ainsi un important défi à l'université. Dans ce contexte, la loi ORE, via l'application Parcours Sup, prévoit de distinguer les bacheliers directement admis à l'université avec un «oui» franc de ceux considérés comme nécessitant un accompagnement spécifique, acceptés avec un «oui si». L'optique de la loi est clairement celle d'une remédiation, fondée sur le principe de lacunes à combler chez ce public particulier. Son application rencontre un certain nombre d'oppositions, et suite à la mobilisation du printemps 2018, cette mesure n'a pas été appliquée uniformément dans les universités françaises.

11 Alors que la montée en puissance du thème de la « difficulté scolaire » peut être perçue comme relevant, au moins partiellement, d'une «psychologisation du social» (Roiné, 2014), le risque est réel de reproduire ce type de cercle vicieux lors de la mise en place de tels dispositifs spécifiques dans l'enseignement supérieur, et de faire porter à la frange du public universitaire la plus éloignée des attentes académiques les conséquences de choix politiques qui les dépassent, en traitant leurs difficultés uniquement comme le reflet de déficiences personnelles. La vigilance vis-à-vis de cet écueil est d'autant plus nécessaire que les études montrent que ce processus d'essentialisation et de stéréotypisation des élèves en difficulté à l'école, est bien à l'œuvre, chez l'ensemble des membres de la communauté éducative, touchant aussi bien les enseignants en formation, les enseignants spécialisés, les psychologues scolaires que les parents d'élèves ou les élèves-mêmes (Desombre et al., 2008). Or, depuis l'expérience fondatrice de Rosenthal et Jacobson (1968) révélant le célébrissime 
effet Pygmalion, et malgré toutes les nuances que des études ultérieures ont permis de préciser, il est admis que cet étiquetage peut avoir des effets auto-réalisateurs (Mingat, 1991, Bressoux et Pansu, 2003; Jussim et Harber, 2005). D'une part, il donne lieu à des comportements et attentes différents à leur égard, même en contexte ordinaire, susceptibles de freiner leur réussite (Potvin et Rousseau, 1993, Good et Brophy, 2000). D'autre part, le processus de stigmatisation joue sur l'image de soi, et ce d'autant plus que les élèves manquent de ressources pour y résister (Madon et al., 1997): en se percevant comme moins capable de réussir que les autres, on a, de fait, moins de chances de réussir. La question de l'image de soi est fondamentale puisqu'elle peut alors fonctionner comme une double peine : la personne n'ayant pas confiance dans ses compétences et ne se sentant pas investie par les autres de la capacité à prendre en main son apprentissage risque de ne pas s'estimer capable de l'auto-accomplissement nécessaire pour tirer bénéfice de l'accompagnement qui lui est proposé (Little, 2010, p. 28). Le grand avantage dont dispose aujourd'hui la pédagogie universitaire à l'heure de mettre en place des dispositifs spécifiques visant à aider les étudiants et étudiantes les plus fragiles est de pouvoir s'appuyer sur les résultats des expériences menées dans les enseignements primaire et secondaire. Cela peut permettre d'éclairer l'ingénierie didactique à partir de la documentation des pratiques antérieures et de leurs effets. Ainsi, les dispositifs temporaires, perçus par l'ensemble des acteurs éducatifs comme transitoires, limitent davantage la stigmatisation qu'une prise en charge longue (Suchaut, 2008). Un autre élément important pour obtenir des effets positifs réside dans le fait que le dispositif ne vienne pas en remplacement de cours qui priveraient les élèves d'enseignement disciplinaires dont ils auraient besoin pour réussir (Mingat, 1991). Plus généralement, il s'agit de s'assurer de la cohérence de ce qui est proposé dans ce temps spécifique vis-à-vis des attentes générales dans le parcours auquel on souhaite que ces personnes restent "accrochées ", cohérence passant nécessairement par une collaboration forte de l'équipe éducative. En effet, la difficulté à faire le lien entre ce qui est travaillé dans cet espace spécifique et les attentes académiques des autres cours est un des facteurs d'échec de ces dispositifs (Conseil national d'évaluation du système scolaire, 2016, Félix et al., 2012). Enfin, les études quantitatives montrent que le taux d'encadrement joue sur l'efficacité des dispositifs (Bressoux et Lima, 2003). Ce dernier point confirme que la question des moyens mis à disposition reste cruciale, même si la réussite des élèves ne suit pas une évolution proportionnelle à celle des financements investis (Suchaut, 2008, Bressoux et al., 2016). Ce décalage peut alors s'expliquer par les choix pédagogiques opérés.

Dans le dispositif CLEFS, nous travaillons en mode projet (Proulx, 2004), en groupe, et insistons sur le soutien socioémotionnel des étudiants et étudiantes accompagnés. Si l'on reprend la typologie des dispositifs proposée par Lescouarch (2011), cela correspond à une double logique mettant l'accent sur le sens aux apprentissages et la remise en confiance. Le mode projet permet de donner du sens aux apprentissages universitaires en les associant à un objectif de réalisation concrète, ici l'organisation d'un événement public. Le travail en groupe permet de limiter les effets de la stigmatisation en sortant de la confrontation individuelle avec la problématique du renforcement de certaines compétences, et en portant à l'échelle du groupe la question de la perception de la capacité à réussir. Enfin, prendre le temps, dans un groupe restreint ad hoc, de créer de la confiance pour explorer les enjeux psychosociaux liés au fait de se sentir à sa place à l'université permet de prendre conscience, avec des personnes partageant une communauté de vécu, des tendances sociales lourdes 
influant sur les trajectoires de vie et de formation, et de mettre en commun les stratégies individuelles et collectives imaginées dans ce contexte commun. Nous faisons ainsi le pari pédagogique que ces processus d'explicitation et de partage peuvent également armer les élèves contre les effets délétères de la stigmatisation sur l'image de soi. L'ambition de ce soutien socioémotionnel dépasse le simple souci de contrecarrer une éventuelle stigmatisation, et cherche à favoriser la construction, chez les personnes accompagnées, d'une image de soi en réussite, notamment en adaptant les objectifs visés et en valorisant chaque progrès réalisé, pour renforcer la perception de soi comme capable d'auto-accomplissement, et ainsi tirer au mieux profit du dispositif. En effet, ce principe de fonctionnement ne se limite pas à la poursuite du grand projet du semestre. Pour chaque activité proposée, la démarche recherchée est de sortir de l'exercice purement académique et de rendre la tâche nécessaire pour la poursuite d'une intention projetée par l'élève, ainsi incité à sortir des postures première, scolaire ou de refus typiques d'un niveau scolaire faible, et à faire l'expérience de postures ludique-créative et réflexive (Bucheton, 2011, cité dans Reverdy, 2017). Les moments de travail portant sur l'écrit n'échappent pas à cette approche d'ensemble, et, dans un contexte didactique fortement tourné vers la collaboration, la réflexivité, et une production publique, ils sont principalement associés à une intention de dialogue. En termes de soutien socioémotionnel, au-delà d'un accompagnement technique vers la littéracie universitaire, il s'agit également de faciliter le rapport à l'écrit académique, qui peut paraître inaccessible à un public peu assuré de sa légitimité à se lancer dans la poursuite d'études supérieures. L'objectif est de mobiliser à la fois des ressources cognitives et psychosociales pour progresser dans l'appropriation de l'écrit universitaire. Ainsi, pour paraphraser la distinction argumenter pour apprendre/apprendre à argumenter, s'intéresser spécifiquement aux compétences de littéracie revient à la fois à lire-écrire pour dialoguer et à dialoguer pour lire-écrire.

\subsection{Une approche triplement dialogique de la littéracie universitaire}

13 De tels fondements théoriques ont mené à une approche triplement dialogique de la littéracie universitaire dans le dispositif CLEFS. Premièrement, cette approche est dialogique, au sens où elle s'appuie sur un style d'enseignement dialogique. Ainsi, tout le dispositif est fondé sur l'interaction et l'échange, et se distingue à ce titre d'un style d'enseignement autoritaire, dont l'incarnation stéréotypée serait le cours magistral, qui repose sur une logique descendante de transmission des connaissances (Scott et al., 2006). Si les recherches de pointe en éducation ne cessent de souligner l'intérêt de l'enseignement dialogique, elles mettent également en avant le fait qu'il présente de réelles difficultés, et qu'enseigner de cette façon nécessite une boite à outils bien fournie (Alexander, 2017). Cela suppose aussi des conditions de travail appropriées, notamment en termes d'effectifs, et une évolution des pratiques des institutions et acteurs éducatifs. Ces derniers facteurs participeraient à expliquer que l'approche dialogique reste minoritaire, et que, lorsqu'elle est utilisée, elle le soit de façon hybride, couplée à des pratiques plus autoritaires (Scott et al., 2006).

14 L'approche développée est également dialogique au sens où elle est associée à des situations didactiques de travail en groupe et/ou cherchant à faire advenir et à exploiter les vertus du conflit sociocognitif (Doise et Mugny, 1981) dans le dialogue entre pairs. On retrouve ici la double entrée de la littéracie en vue du dialogue et du 
dialogue en vue de la littéracie. Le style d'enseignement dialogique qui caractérise le dispositif à l'échelle macroscopique est ainsi incarné dans des activités d'appréhension de la lecture et de l'écriture dialogiques, à une échelle mésoscopique. Une telle approche est fondée théoriquement sur une conception de la littéracie comme pratique socialement située fondamentalement communicative (Hamilton et Barton, 2001), et a donné lieu à des résultats empiriques concluants (Rojas-Drummond et al., 2017).

Enfin, l'approche dialogique renvoie également à des pratiques à l'échelle microscopique, à savoir à l'intérieur même des écrits reçus ou produits. Il s'agit ici d'un dialogisme proche du sens bakhtinien (Todorov, 1981), qui a trait à la façon dont un texte peut être construit en intégrant plusieurs " voix » et points de vue avec lesquels l'auteur entre en résonance, qu'il s'agisse de s'appuyer sur elles, de les soutenir, ou de s'y opposer. Si de nombreux types d'écrits peuvent présenter cette caractéristique, elle constitue un ingrédient important du genre académique, ne serait-ce que par la pratique de cadrage théorique associé à l'introduction de tout objet d'étude. Des études empiriques ont montré que cette capacité à comprendre et manier un discours dialogique peut également être travaillée collectivement : après une discussion en petit groupe constructive, les élèves parviennent à complexifier leurs arguments en intégrant les points de vue des autres et en anticipant d'éventuels contre-arguments (Albe, 2006; Polo et al., 2016).

\section{Contexte institutionnel et didactique}

Avant de montrer comment les activités concrètes consacrées à la littéracie universitaire au sein du dispositif CLEFS procèdent de cette approche dialogique, il convient de préciser le contexte institutionnel et didactique dans lequel elles sont développées.

\subsection{Des moyens humains : cadre institutionnel et collaboration entre composantes}

Des moyens importants ont été alloués à la mise en place d'un "parcours de remédiation » expérimental, visant à préfigurer quel traitement spécifique l'université pouvait proposer aux bacheliers accueillis avec la mention « oui si » née de la loi ORE. Dans le contexte d'une mobilisation, au sein de l'université, contre cette réforme, la présidence de l'Université Lyon 2 affiche la volonté de s'appuyer sur l'expertise de la recherche en éducation, pour créer un dispositif innovant, en fléchant ces moyens sur la création de deux emplois pérennes en septembre 2018: un support d'enseignantchercheur en sciences de l'éducation et un poste d'ingénieur pédagogique, tous deux rattachés à l'Institut des Sciences et Pratiques de l'Education et de la Formation (ISPEF). Plusieurs mois avant leur recrutement, un certain nombre de rencontres sont organisées, conviant pêle-mêle la vice-présidente en charge de la formation et de la vie étudiante, les responsables de plusieurs portails ${ }^{4}$, les coordinateurs des enseignements de méthodologie universitaire, le directeur de l'ISPEF, la directrice du laboratoire de Sciences de l'Education de Lyon 2, Education, Cultures et Politiques, et d'autres membres de l'ISPEF volontaires pour participer à la réflexion. Quelques lignes directrices sont esquissées : mettre l'accent sur une pédagogie active en mode projet visant la réalisation d'un événement public; fonctionner en effectifs réduits (groupes de 
10-12), sur quelques portails, pour cette phase expérimentale; associer à l'enseignement à la fois du personnel spécialisé dans les disciplines concernées et des membres de l'ISPEF, pour leur expertise pédagogique, à hauteur de $63 \mathrm{~h}$ par groupe; commencer dès le premier semestre, pour tenter de retenir les personnes qui décrochent avant les premiers examens; assurer un lien important avec les composantes via la désignation d'un interlocuteur privilégié par portail rémunéré à hauteur de $21 \mathrm{~h}$ ainsi que par la mise en place d'un tutorat assuré par des masterants et masterantes des disciplines concernées; principe de volontariat ${ }^{5}$. Ce dernier choix procède d'un positionnement philosophique fort, qui préfère à la prescription d'un dispositif d'aide obligatoire une véritable posture d'accompagnement, qui n'impose pas un horizon mais chemine auprès de la personne accompagnée vers « ses buts " propres (Beauvais, 2004, p. 101), et ce alors que le contexte plus général est celui de la mise en place par l'institution d'une réforme descendante que ses personnels sont chargés d'appliquer. On est ici dans une situation de tentative collective de "retournement » : "un retournement de l'accompagnement comme " aide " en " ressource ", un retournement de l'accompagnement comme " commande » à exécuter en "levier » à saisir » (Paul, 2010, p. 20).

Depuis la rentrée 2018, la présence d'une ingénieure pédagogique se consacrant à plein temps au parcours CLEFS ainsi que l'arrivée d'une maitresse de conférences responsable du dispositif (auteure de cet article) ont nettement accéléré les choses, dans un contexte temporel très contraint visant le début de la première expérimentation dès la rentrée des vacances universitaires de la Toussaint. Une demande de financement externe parallèle concernant la création d'un MOOC associé à ce dispositif, infructueuse, a par ailleurs finalement abouti à une dotation en interne sur les fonds de l'université, permettant d'envisager sa mise en œuvre dès le second semestre universitaire, début 2019. Un premier positionnement important a été de trouver une dénomination traduisant mieux la démarche retenue que "parcours de remédiation ", c'est ainsi qu'est né le dispositif CLEFS.

19 Au plan institutionnel, afin d'éviter de priver les bénéficiaires du dispositif CLEFS de cours disciplinaires fondamentaux sans toutefois surcharger leur emploi du temps ${ }^{6}$, une nouvelle UE Transversale a été créée, où le module CLEFS vient remplacer les travaux dirigées (TD) de Méthodologie et de Projet Personnel et Professionnel, pour un coefficient représentant 3 ECTS. Pour l'instant, l'ensemble du dispositif ne concerne qu'un nombre très réduit de personnes: 12 places ont été ouvertes par portail. Les quatre portails concernés par la phase expérimentale du dispositif présentent une relative proximité disciplinaire : Institutions et Société (IS); Médias, Cultures, Sociétés (MCS); Sciences Sociales (SS); Temps Territoire Société (TTS). Un canevas de séquence pédagogique commun a été conçu, puis discuté et adapté pour chaque portail en fonction des spécificités disciplinaires et des possibilités concrètes de collaboration. La première séance de cours présentiel de $2 \mathrm{~h}$ réunit la personne référente du dispositif dans le portail, la personne suivant le groupe pour la part des enseignements dévolus à l'ISPEF, et, pour la première heure, les tuteurs et tutrices, qui font à cette occasion connaissance des personnes qu'ils accompagnent. L'ensemble de ces personnes est également réuni pour un bilan de $2 \mathrm{~h}$ en fin de dispositif. Dans l'intervalle, les bénéficiaires du dispositif CLEFS reçoivent au total $53 \mathrm{~h}$ de cours hybride $(32 \mathrm{~h}$ en présentiel à raison de $4 \mathrm{~h}$ par semaine sur 7 semaines puis $2 \mathrm{~h}$ par semaine pendant les 2 semaines d'examens terminaux; $12 \mathrm{~h}$ à distance à raison de $2 \mathrm{~h}$ par semaine sur 6 
semaines; $3 \mathrm{~h}$ consacrées à la préparation et la réalisation de l'évènement public et $2 \mathrm{~h}$ de bilan).

\subsection{Un accompagnement triple : cours présentiel, travail à distance et tutorat}

20 L'ensemble des cours prévus dans le dispositif a été pensé dans une articulation entre une partie présentielle, principale, et une partie complémentaire de travail à distance. Le travail en face-à-face est articulé autour du projet en petits groupes, tandis que le contenu de la formation à distance vise à aider les élèves à s'organiser individuellement vis-à-vis de leurs échéances personnelles, et à permettre un suivi personnalisé de leurs écrits. Cet ensemble hybride est composé des six modules suivants :

21 1. Se positionner dans le groupe CLEFS et dans l'université

2. Qu'est-ce qu'étudier

3. Entrer dans un projet en groupe

4. Se documenter

5. Appréhender un objet d'étude

6. Communiquer publiquement l'état d'une réflexion et les savoirs afférents.

Le premier module a pour but de familiariser les étudiants et étudiantes avec la démarche pédagogique du dispositif, et de leur accorder un temps spécifique pour réfléchir au sens de leur engagement dans leur parcours universitaire, notamment via des jeux théâtraux. C'est également un moment important pour construire de la confiance dans le groupe de TD. Dans ce module, sont explicitement abordées à la fois les multiples fonctions sociales de l'université fonctionnant comme des contraintes sur les trajectoires individuelles, et les ressentis personnels au moment de chercher à y trouver une place. L'enjeu est de prendre en compte la problématique de l'image de soi dans la réussite universitaire, tout en évitant une psychologisation excessive qui amènerait à ne reconnaître que des causes internes à la difficulté scolaire. Cette étape est essentielle pour limiter le risque d'effets néfastes associés à une stigmatisation, d'autant que l'ensemble du dispositif, par sa nature même, ne peut proposer ensuite qu'un travail sur ces fameuses causes internes de la difficulté universitaire : motivation, perception de sa capacité à réussir, compétences techniques et habiletés cognitives (et non sur des causes externes telles que le fait de devoir travailler pour financer ses études, les problématiques de logement, etc.). Il s'agit également d'un temps important de rencontre et de construction de la relation d'accompagnement et de «coaccompagnement ", avec les pairs, les enseignants et enseignantes, les tuteurs et tutrices. C'est en effet dès le début du parcours qu'est travaillée la dimension de " socialisation universitaire ", entendue à la fois comme familiarisation aux normes et valeurs de comportement à l'université et comme établissement de liens permettant de s'identifier au groupe des étudiants ou étudiantes.

Le second module propose d'aborder plusieurs compétences fondamentales (organisation du travail, méthodologie, techniques de lecture et d'écriture) pour réussir à l'université et comprendre le métier d'étudiant via un travail collectif d'enquête. Deux des quatre volets de l'enquête portent sur la littéracie universitaire : à savoir comment les personnes plus avancées dans leur cursus, les personnels académiques et les professionnels issus des mêmes filières, se sont saisis, dans leurs pratiques concrètes, de l'écrit académique. Plutôt que de prescrire une façon de faire, il s'agit 
d'accompagner les élèves vers la découverte de multiples techniques développées par leurs aînés, autant d'exemples et de pratiques visant à leur fournir un vaste éventail de possibilités à partir duquel se fabriquer de manière ad hoc une «méthodologie de l'apprentissage » adéquate (Holec, 1991).

Les modules 3-4-5 correspondent à différentes facettes du travail en mode projet réalisé sur le contenu à transmettre lors de l'évènement public final. Ils peuvent prendre des formes relativement différentes selon les portails, certains privilégiant le travail de terrain, d'autres, le travail sur corpus documentaire, en fonction des disciplines. Ces modules peuvent être enseignés à la suite les uns des autres, ou en alternance. L'enjeu est de toute façon de faire comprendre l'intérêt de se documenter pour appréhender un objet d'étude, et de faire vivre l'expérience d'une démarche de projet. Ainsi, un des objectifs est que les étudiants et étudiantes saisissent l'apport d'un va-et-vient, d'un dialogue entre lectures académique et travail empirique.

Le module 6 a directement trait à la réalisation d'une communication publique du travail accompli, passant par une préparation écrite, même si des formats d'évènements variés ont été retenus par les quatre portails: exposition guidée, conférence-débat, organisation d'une table-ronde, disputatio (mise en scène argumentative autour d'une controverse théorique). Les modules 1 et 2 sont plutôt assurés par des enseignants de l'ISPEF, référents pédagogiques, tandis que les modules 3 à 5 relèvent davantage des enseignants spécialistes des disciplines des portails. L'ensemble des intervenants collabore autour du module 6. La pédagogie par projet se prête particulièrement bien à l'entrée, pour les enseignants, dans une posture d'accompagnement, qui suppose de cheminer avec les personnes vers les objectifs et actions vers lesquelles elles avancent, dans une perspective de "co-conscientisation " et de « co-construction » de leurs projets (Beauvais et Haudiquet, 2012, p. 169).

Outre des consignes spécifiques accompagnant ce travail présentiel, le cours à distance comprend un carnet de bord individuel portant sur l'organisation du travail personnel et l'expérience subjective de la vie universitaire. Les élèves sont amenés à le nourrir très régulièrement, puisque les deux heures hebdomadaires de formation à distance prévues sur 6 semaines sont séquencées en 4 périodes de 30 minutes du lundi au jeudi, favorisant un rythme de travail régulier. Le recours à cet outil vise à favoriser la posture réflexive, indispensable à la construction de leur autonomie dans l'apprentissage (Little, 2010, p. 29).

27 Enfin, en parallèle, les personnes qui intègrent le dispositif bénéficient d'un tutorat, réalisé par des élèves de master rémunérés, prévoyant $1 \mathrm{~h}$ de suivi hebdomadaire par groupe de trois, pendant 10 semaines. L'objet du tutorat ne se limite pas au soutien du projet élaboré dans le cadre du groupe CLEFS, et doit permettre aux tutorés d'aborder n'importe quel aspect de leur travail universitaire du semestre. Conformément aux lignes directrices du dispositif, les tuteurs sont spécialement formés pour cette mission par la responsable du dispositif, et une partie de la formation porte explicitement sur le soutien socioémotionnel des personnes accompagnées, abordé sous l'angle de mises en situation et d'outils concrets (travail sur les « réussites", de petites avancées à de plus grands progrès, entraînement à réagir face à des personnes exprimant un mal-être, etc). L'enjeu de ce tutorat est également de compléter l'accompagnement par les enseignants et enseignantes d'un accompagnement plus horizontal, du fait de la moindre distance sociale qui sépare les personnes suivies de leur tuteur ou tutrice. En effet, une juste posture d'accompagnement suppose la recherche incessante de 
l'affirmation d'une égalité (existentielle, de nature, entre humains, s'incarnant dans la prise en compte de l'accompagné comme personne), sur fond d'inégalité (des puissances, voire de statut entre accompagnant et accompagné, à un moment donné, dans un champ donné) (Paul, 2004, p. 139-140).

\subsection{Formation des premiers groupes CLEFS : du public visé aux personnes inscrites}

Faire le choix de ne pas s'appuyer sur le classement en « oui si » réalisé dans Parcours Sup pour former les groupes CLEFS impose une véritable réflexion sur le ciblage et le repérage du public accompagné. L'enjeu n'était pas de reproduire sous une autre forme un soutien déjà apporté par ailleurs à certains publics spécifiques: handicap, intégration des personnes étrangères en échange universitaire, réorientation. Ce qui a fait consensus était plutôt la volonté d'innover, au plan pédagogique, pour accompagner des élèves " risquant de décrocher ", et ce dès le premier semestre, mais néanmoins ayant une véritable motivation pour le cursus choisi et étant suffisamment proches des attentes académiques pour parvenir à accrocher aux études universitaires. Cependant, nous avions conscience que les causes d'un tel décrochage sont multiples, et dépendent autant des personnes concernées que de leur environnement (Tessier et Schmidt, p. 571). En tant que processus, le décrochage peut de plus advenir plus ou moins progressivement, sur une période temporelle plus ou moins longue, et implique, au rang des facteurs actuellement documentés, non seulement l'inadéquation des méthodes de travail aux attentes universitaires, mais également l'orientation "par défaut ", et la difficulté à articuler travail universitaire et activités extra-académiques, notamment une activité salariée parallèle (Beaupère et Boudesseul, 2009).

Sur cette base, le repérage des bénéficiaires du dispositif a été organisé en deux volets, centrés sur les compétences qu'il peut permettre de renforcer, et, en creux, sur l'écartement de difficultés d'autres natures (réorientation, public allophone). Le premier volet, qualitatif, s'est appuyé sur l'expertise des personnes chargées de TD de méthodologie et sur leur connaissance des étudiants et étudiantes. En effet, entre la rentrée universitaire et l'interruption pédagogique de la Toussaint 2018, toutes les personnes inscrites en L1 ont suivi ces TD, qui sont l'occasion de faire le point sur d'éventuelles difficultés méthodologiques. Les responsables de ces TD ont reçu une fiche les incitant à repérer d'éventuelles fragilités chez leurs étudiants et étudiantes avec des indicateurs concrets à observer. De plus, des questionnaires papier individuels ont été passés dans tous ces groupes de TD. Les élèves étaient amenés, via cette enquête, à déclarer d'éventuelles difficultés, abordées à travers des situations concrètes. C'était aussi l'occasion de clarifier leur projet professionnel, et d'expliciter leur situation actuelle, notamment vis-à-vis d'éventuels projets de réorientation, et de contraintes rendant leur participation au dispositif plus difficile (volume de travail extra-universitaire important, lieu de vie très éloigné du campus). Une question portait également sur leur intérêt pour intégrer le parcours CLEFS si on le leur proposait. Enfin, la dernière question, plus ouverte, leur laissait une opportunité de s'exprimer davantage, nous permettant d'évaluer leur degré d'aisance à l'écrit, tout en nous renseignant sur leur ressenti par rapport à cette nouvelle rentrée. Etant donné les délais de mise en place très courts du dispositif pour ce premier semestre, la priorité a été donnée au repérage qualitatif. Ainsi, les responsables des TD ont été chargés de transmettre les questionnaires remplis par leurs élèves tout en indiquant lesquels 
semblaient, à leur avis, prioritaires pour en bénéficier, dossiers que nous avons traités avant les autres.

Un certain nombre d'imprévus a néanmoins été à l'origine d'un relatif décalage entre le public initialement ciblé, ainsi que la méthode de repérage a priori envisagée, et la constitution finale des listes d'inscription dans le dispositif CLEFS. Une première difficulté a résidé dans un retard important dans la passation et le recueil des questionnaires, ainsi que dans la désignation par les responsables de TD de personnes particulièrement susceptibles d'intégrer le parcours. Nous avons privilégié les profils à la fois repérés par les responsables de TD et ayant clairement exprimé, par écrit, le souhait d'intégrer le parcours, dès lors que leurs questionnaires ne faisaient pas état d'un problème clair d'orientation, de maittrise du français pour les personnes dont ce n'était pas la langue maternelle, ou d'incompatibilité administrative. Des listes de 12 personnes retenues et de 12 personnes sur liste complémentaire, ont ainsi été réalisées. Lorsque nous n'atteignons pas 24 personnes, nous avons considéré les dossiers de personnes ayant exprimé un « oui » quant à la volonté de participer éventuellement au dispositif, mais non repérées par leur enseignant ou enseignante, puis les personnes ayant coché « peut-être » pour cet item, en priorité si les responsables de TD les avaient mentionnées.

\section{L'accompagnement à la littéracie dans le dispositif CLEFS}

Bien que de véritables aspects de méthodologie et de littéracie universitaire soient abordés dans ce dispositif, le parti pris retenu consiste, en quelque sorte, en ce que le référent d'un des portails concernés a appelé une "déméthodologisation» de l'enseignement des compétences ciblées. Ainsi, plutôt qu'un enseignement formel de façons de faire, et en particulier, pour ce qui nous intéresse ici, de façons de lire et d'écrire, il s'agit de mobiliser l'écrit pour servir un projet précis, impliquant une intention de dialogue. L'enjeu est de mettre les élèves en situation de ne pas pouvoir faire l'économie d'une appropriation de l'écrit universitaire pour parvenir à leur objectif. La première étape dans l'accompagnement de ce public consiste à faciliter un rapport socioaffectif à la lecture et à l'écriture parfois difficile en général. Dans cette perspective, les actions de lire et d'écrire sont envisagées de façon fondamentalement multimodale, des supports didactiques et canaux de communication variés étant utilisés : texte mais aussi image, graphique, tableaux, film, documents sonores. Dans un premier temps, l'enjeu principal est de permettre l'autonomisation de ce public à travers une modification de leur rapport à la lecture et à l'écriture, pouvant prendre des formes d'écrit diverses. Une seconde étape consiste à accompagner ce public vers l'appréhension de l'écrit académique, notamment en lui faisant toucher du doigt le sens et les fondements épistémologiques de ce genre. Par exemple, c'est en initiant les élèves à une démarche critique et scientifique que l'on cherche à leur faire comprendre l'intérêt de citer précisément ses sources, de rendre compte de lectures mais aussi de les situer vis-à-vis d'autres approches, de construire la sienne propre, d'argumenter cette position. Plus précisément, cette approche dialogique de la littéracie s'incarne dans des pratiques de lecture et d'écriture concrètes prévues dans ce dispositif. 


\subsection{Lire pour dialoguer et dialoguer pour lire} lire. Lors de la deuxième séance, qui revient sur une question fondamentale « Pourquoi l'université? L'université, pour quoi?», quatre documents différents sont étudiés, par groupe de trois, qui relèvent de genres différents et qui éclairent, du point de vue d'une pluralité d'acteurs, les fonctions sociales de l'université. L'exercice, relativement classique, consiste à extraire des informations du document, pour présenter ensuite oralement, en groupe, en quoi il éclaire la question. Sur ce dernier point, on bascule dans le lire pour dialoguer : l'objectif est bien de mieux réfléchir au problème posé à partir des éléments apportés par les différentes lectures. A cette occasion, un travail réflexif sur les stratégies d'écriture en vue de la mise en commun est ensuite opéré : on regarde les différents supports à l'expression orale produits et on les compare, on fait le lien entre la prestation orale de chaque personne et les formes de notes sur lesquelles elle s'est appuyée (texte entièrement rédigé, idées principales sous forme de mots-clefs, notes manuscrites ou sur écran, etc.).

Par la suite, l'essentiel de l'approche de la lecture relève du lire pour dialoguer, qu'il s'agisse de dialoguer avec les autres personnes du cours, avec un objet d'étude, ou avec le public à qui est destiné l'évènement final en cours de préparation. Les séances 2 et 3 de travail à distance sont organisées autour de la lecture de textes courts et simples (issus de manuels de lycée, cf. par exemple l'annexe 1). Le premier évoque le ressenti inconfortable lié à une concurrence entre socialisations universitaire et familiale. L'exercice consiste à produire des réponses argumentées à quelques questions, qui guident les étudiants et étudiantes vers une interprétation sociologique de ce ressenti. Ces écrits sont ensuite travaillés en vue d'un dialogue à distance avec les autres membres du groupe. Le second texte porte sur le sentiment d'incompétence en politique. L'exercice consiste alors à lister des questions suscitées par sa lecture puis à identifier plus spécifiquement celles qui offrent matière à discussion.

Il s'agit également de lire pour dialoguer avec l'objet étudié dans le projet entrepris, pour réussir à l'interroger et à mieux comprendre ce qu'il peut nous « dire ». Ainsi, les élèves sont amenés à réaliser un travail de recherche bibliographique pour problématiser leur approche d'un terrain (exploration d'un espace social pour le portail SS, par exemple) ou d'un corpus (images violentes pour le portail MCS, par exemple). Un double accompagnement est ici proposé. Au plan organisationnel, les étudiants et étudiantes sont accompagnés dans leur recherche documentaire, à la fois de façon très pragmatique pour apprendre à utiliser la bibliothèque universitaire, et dans la planification et mise en œuvre concrète de leurs lectures avec plusieurs séances à distance les aidant à se repérer parmi les références trouvées, les hiérarchiser, et à produire des formes d'écrit permettant d'avoir des traces des documents lus. Au plan cognitif, ce travail sur la lecture en vue d'un dialogue avec l'objet étudié donne lieu à deux séances de cours présentiel dédiées à l'arpentage ${ }^{7}$ collectif d'un document académique de référence sur la grande thématique explorée, où les formules, concepts et thèses sont explicitées et discutées. Ces séances sont plutôt assurées par des spécialistes. Elles visent à décrypter collectivement un texte fondamental et en tirer des éléments utiles aux projets développés par les différents groupes. En tant que méthode, l'arpentage comme outil pédagogique s'appuie sur la dynamique du dialoguer

Revue internationale de pédagogie de l'enseignement supérieur, 36(2) | 2020 
pour lire. Cependant, il s'agit bien ici non seulement de comprendre le document luimême, mais aussi de pointer son intérêt pour l'intention créative collective.

En effet, l'enjeu final est bien de lire pour dialoguer avec un public, et certaines formes d'évènement final imaginées supposent des phases de lecture précédant les échanges. Par exemple, le groupe CLEFS pour portail TTS a choisi pour évènement final la mise en scène d'une disputatio, qui suppose la récitation d'un texte préparé en amont. Une autre forme de lecture publique consistera par exemple, pour le portail MCS, à présenter et décrypter des images au public avant d'en proposer des analyses des points de vue artistique, académique et journalistique.

\section{2. Écrire pour dialoguer et dialoguer pour écrire} espaces-temps, toujours en vue du partage d'écrits avec autrui, et principalement dans la formation à distance. Ainsi, les réponses argumentées à propos du texte abordé dans la deuxième séance de travail à distance sont relues par l'enseignant, qui suggère des modifications, puis corrigées par leur auteur, avant d'être visibles sur l'espace numérique de travail commun, afin d'être discutées par d'autres. De même, quelques séances de travail à distance sont dédiées à un moment d'écriture créative en lien avec le thème exploré. Il s'agit à la fois de faire émerger des représentations sur la question étudiée, et de chercher à susciter un rapport ludique aux mots et un véritable plaisir dans l'expérience d'écriture. Le premier jet, là aussi, est visé par l'enseignant ou l'enseignante, et réécrit par son auteur avant d'être rendu visible aux autres. A ce stade, les retours portent principalement sur la correction orthographique et syntaxique du texte, et c'est ultérieurement, lors de la mise en commun en présentiel qui suit la publication dans le groupe, qu'un travail méta-discursif de description comparative des différentes productions a lieu, se rapprochant du travail sur les représentations du résumé, par exemple, étudié par Alves (2005). Il s'agit d'acquérir des bons réflexes vis-à-vis de pratiques d'écriture intermédiaires, qui ne présentent pas les caractéristiques du genre académique, mais qui sont 
indispensables à la réussite universitaire : fiches de lecture, traces d'une démarche d'enquête, notes en vue d'une présentation orale, etc. Il s'agit de systématiser le recours à ces formes d'écrits non dans une posture scolaire, mais comme ressources pour la mutualisation des savoirs construits, au sein du petit groupe de travail, puis pour mettre en commun les résultats du groupe avec l'ensemble des membres du TD. D'ailleurs, l'accompagnement proposé est fondé sur une progression de ces écrits intermédiaires vers des formes se rapprochant davantage du genre académique. Ainsi, lors de la seconde séance en présentiel, un retour réflexif est réalisé sur la façon dont les élèves s'y prennent pour préparer, via quelques notes écrites, la restitution concernant le document étudié. En fin de parcours, lors de la restitution des travaux de groupes en TD, c'est via un poster qu'ils doivent structurer leur prise de parole. Pour le portail SS, cet « écrit-pour-l'oral » prendra la forme de panneaux d'exposition lors de l'évènement final. Il n'en demeure pas moins que ces écrits intermédiaires gardent une utilité en complément des supports écrits présentés à l'auditoire. La démarche n'est donc pas tant un "passage de l'écrit à l'oral » ou vice-versa qu'une complémentarité fonctionnelle de l'écrit et de l'oral.

En effet, l'ensemble du dispositif est conçu pour chercher à donner du sens aux compétences travaillées, et la production d'écrit participe de cette démarche. L'objectif est de mettre les étudiants et étudiantes en situation de faire l'expérience de différentes fonctions de l'écriture, pour s'approprier ces pratiques comme un répertoire d'outils à leur disposition dans lequel puiser pour gagner en autonomie dans leur travail universitaire. Il s'agit bien sûr de sa fonction mémorielle qui sauve des pensées/lectures de l'oubli, une séance de travail à distance étant consacrée justement à la question du partage de réflexions lues ou émergeant du travail empirique, nécessaire à la conduite d'une recherche en groupe. La matérialité de l'écrit servant, comme référence, à la représentation de l'état d'un travail ou d'un accord collectif sur sa planification, est également abordée, via un pad en ligne où sont mises en commun les traces de l'enquête réalisée par chaque membre du groupe en parallèle, ou encore la rédaction collective d'une grille d'enquête. Dans cet état d'esprit, les élèves sont également amenés à écrire puis partager le compte rendu d'un évènement public auquel ils ont assisté, similaire à celui à organiser. Une autre fonction de l'écrit mise en lumière est le potentiel de représentation synthétique d'objets complexes offert par des modalités écrites non textuelles ou co-textuelles (schémas, dessins, tableaux). Ainsi, une activité menée en TD présentiel, sur le modèle des DVDP (Discussions à Visée Démocratique et Philosophique) (Gagnon, 2005), se termine par le recours à une métaphore qui peut passer par le dessin, et elle donne lieu à un travail à distance consistant à réaliser une carte mentale des différents argumentaires déployés au cours de la discussion. Enfin, l'écriture est rendue indispensable comme support de la réflexivité dans le dispositif. Effectivement, les élèves sont souvent appelés à retravailler un premier écrit, non seulement en termes de correction de la langue, mais également pour avancer dans leur raisonnement. Ainsi, la deuxième séance en présentiel inclut un brainstorming sur les fonctions sociales de l'université, où chaque personne est invitée à lister des idées, qui sont ensuite regroupées pour former des grandes catégories. De même, lors de la troisième séance de travail à distance, un exercice consiste à lister toutes les questions suscitées par la lecture d'un texte, puis à les classer. Après le travail d'enquête réalisé auprès de personnes ayant validé la licence à laquelle elle se destine, une séance à distance amène chaque personne à retravailler sur ses propres atouts pour réussir et ses points de vulnérabilité, en 
complétant un bilan personnel sous forme d'un écrit multimodal (dessin et texte) en intégrant les points mis à jour par l'enquête. L'expérience de pouvoir se relire et de relire les arguments des autres membres du groupe CLEFS a posteriori et avec davantage de temps que dans le feu de l'échange oral, pour les évaluer et construire son propre argumentaire écrit, fait aussi partie de la prise de conscience de ce potentiel réflexif offert par l'écriture.

Plus généralement, l'argumentation est au cœur de l'accompagnement proposé au sein des cours CLEFS. Elle est présente comme stratégie didactique dialogique fondée sur le conflit sociocognitif, mais aussi comme orientation inhérente à toute forme de discours produit sur la thématique abordée, puisque, conformément à l'orientation disciplinaire des portails impliqués, les questions choisies sont controversées. La nécessité de fonder un point de vue sur de telles questions pour le rendre, a minima crédible, au mieux convaincant, aux yeux des autres, fait partie intégrante du genre académique. Un travail important sur les compétences argumentatives est donc mené, à l'oral, en Communauté de Recherche Philosophique pour renforcer les "habiletés de pensée " (Lipman, 2003), comme à l'écrit, via la carte mentale synthétique de cette discussion, mais aussi pour toute forme d'écrit sur une question ouverte, qui doit expliciter les raisons sur lesquelles se fonde la thèse défendue. Là encore, il y a un écho entre l'approche dialogique de la lecture et de l'écriture, puisque les textes travaillés le sont également principalement sous l'angle argumentatif: il s'agit d'identifier la thèse soutenue, les arguments employés pour ce faire, et comment ils dialoguent avec d'autres interprétations théoriques et/ou avec les données issues du travail empirique. Utiliser la clef argumentative comme prisme d'appropriation de l'écrit académique permet de ne pas s'en tenir à un enseignement formel d'un style académique (niveau de langage, structure argumentative), mais de faire comprendre les enjeux substantiels associés à certains codes du genre (donner ses sources, citer précisément, expliciter les méthodes, etc.). Cette approche a l'avantage de ne pas désolidariser la littéracie universitaire de ses fondements épistémologiques et de respecter les compétences qui en relèvent comme pratiques cognitives et non comme simples figures stylistiques. $\mathrm{Au}$ sein du dispositif CLEFS, l'écriture signifie également, comme c'est souvent le cas en contexte éducatif, une forme d'institutionnalisation didactique d'un discours. Ce que l'individu ose écrire pour le mutualiser au sein de son groupe représente une sélection de ce qu'il a appris, déjà retravaillée dans une forme lui semblant appropriée à ce contexte; ce que le petit groupe communique par écrit au grand groupe est déjà d'un autre registre; et ce qui sera finalement utilisé comme support de l'évènement public sera issu d'une troisième étape de transformation en vue de ce troisième niveau d'institutionnalisation. Mettre l'accent sur l'argumentation, c'est chercher à ce que ces opérations de sélection-transformation soient motivées par la recherche d'arguments les plus robustes possibles, selon le registre argumentaire approprié au contexte concerné. Il serait naïf d'imaginer que la personne responsable du TD soit extérieure à ce processus. En effet, la perception que ses élèves ont de ses attentes structure sans doute leurs choix au cours de ces trois vagues d'institutionnalisation - et ses retours participent eux-mêmes à l'institutionnalisation de raisonnements plutôt que d'autres. Cependant, tout l'enjeu de cette formation est de favoriser l'intériorisation de critères d'évaluation et d'auto-évaluation adéquats aux normes académiques, afin qu'ils gagnent en réflexivité, et partant en autonomie, dans leur travail universitaire. 


\section{Conclusion}

42 Les objectifs portés par le dispositif CLEFS, visant à accompagner des étudiants et étudiantes de L1 repérés comme ayant des difficultés pour accrocher aux études supérieures et réussir leur entrée à l'université, sont relativement ambitieux. D'importants moyens permettent des conditions d'enseignement rares à l'université : 12 personnes maximum par groupe de TD, tutorat, triplement du volume horaire dédié aux méthodologie et littéracie universitaires et au développement de l'esprit critique et scientifique. Le choix pédagogique consistant à renforcer ces compétences via une démarche globale de projet collaboratif rend difficile d'isoler ce qui relèverait spécifiquement de la littéracie universitaire et n'impliquerait pas d'autres savoirs et savoir-faire. Cependant, ce contexte est propice à une forme d'accompagnement à la littéracie universitaire qui rend les étudiants et étudiantes acteurs et actrices de leurs apprentissages, en insistant sur le sens du recours à la lecture et à l'écriture à l'université plutôt que sur des recettes à appliquer en la matière. Ce sens réside principalement dans la nécessité de dialoguer pour apprendre et raisonner; dialogue avec les pairs, avec un objet d'étude, avec la littérature existante sur le sujet abordé, avec le public à qui on destine son travail. Ainsi, au cours du parcours CLEFS, si l'on dialogue parfois pour s'entraider dans l'appropriation de l'écrit académique, on lit et on écrit beaucoup pour dialoguer. L'accompagnement à la lecture et l'écriture proposé est également socioémotionnel: il s'agit de faciliter le rapport affectif à l'écrit. En particulier, «l'entrée en écriture », constitue d'autant plus une épreuve identitaire que les personnes doutent de leur légitimité ainsi soumise au jugement d'autrui (Paul, 2014), ce qui est certainement le cas de personnes adoptant une image d'eux-mêmes comme étant "en difficulté", passage nécessaire présidant à leur entrée volontaire dans le dispositif. Cependant, c'est justement parce qu'elle implique l'image de soi que l'écriture peut, dès qu'elle s'accomplit, avoir un effet de réassurance émancipatoire (Paul, 2014, p. 29).

A ce stade où la conception du dispositif est à peine achevée, et où son expérimentation débute, il est toutefois très difficile de se prononcer sur sa capacité à tenir ses promesses. Le recrutement s'est écarté du chemin prévu, ce qui ne sera sans doute pas sans incidence sur les effets que l'on peut en espérer. A cette occasion, nous avons été confrontés à plusieurs personnes qui avaient été si bien repérées comme susceptibles d'avoir des difficultés à accrocher aux études qu'elles avaient déjà décidé de ne pas revenir à l'université. Cela dit, cette difficile adéquation entre public visé et public accueilli est une problématique classique des dispositifs d'accompagnement (Suchaut, 2008; Duru-Bellat, 2002). En tout état de cause, un important travail de documentation de cette phase d'expérimentation est actuellement en cours. Une analyse plus approfondie des questionnaires permettra sans doute de clarifier les besoins des étudiants et étudiantes se déclarant «en difficulté ». Des entretiens individuels et focus groups sont également prévus en milieu et fin de parcours, en vue d'une évaluation qualitative du dispositif. Enfin, les résultats académiques des personnes accompagnées au cours de leur licence fourniront également une indication sur la capacité du dispositif CLEFS à effectivement ouvrir la porte de la réussite. Etant donné que l'ensemble ne dure que 10 semaines, le devenir de cette première promotion sera également instructif sur le plan de l'objectif d'autonomisation des pratiques d'apprentissage. Après avoir bénéficié sur un temps court d'un accompagnement assez 
intensif, l'objectif est que ces étudiants et étudiantes puissent ensuite être assez autonomes pour réussir à l'université dans les conditions d'encadrement habituelles. Plus largement, cela renvoie à la problématique de l'évaluation d'un tel dispositif. S'il est possible, par exemple, d'avoir une idée de la progression des élèves en écriture en comparant leurs productions finales avec leurs premiers jets, il est délicat d'estimer leur niveau d'autonomie face à la lecture d'un document académique en général, qui suppose une capacité à transférer les méthodes acquises à d'autres sujets. A bien des égards, la littéracie, en tant qu'ensemble de pratiques, renvoie à un horizon vers lequel tendre, et recouvre des compétences qu'il est possible de perfectionner de la L1 jusqu'au doctorat et au-delà.

\section{BIBLIOGRAPHY}

Albe, V. (2006). Procédés discursifs et rôles sociaux d'élèves en groupes de discussion sur une controverse socio-scientifique. Revue française de pédagogie, 157, 103-118.

Alexander, R. J. (2017). Towards Dialogic Teaching : rethinking classroom talk (5 édition). Dialogos.

Alves, J. A., et da Mata, M.A. (2005). A escrita de resumos na formação inicial do professor de língua portuguesa : movimento de aprendizagem no espaço da sala de aula. Dans A. B. Kleiman et M. de L. M. Matencio (dir.), Letramento e formação do professor : práticas discursivas, representações e construção do saber (p. 181-202). Mercado de Letras. http://portal.pucminas.br/imagedb/ mestrado_doutorado/publicacoes/PUA_ARQ_ARQUI20121016143952.pdf

Asterhan, C. S. C. (2013). Epistemic and interpersonal dimensions of peer argumentation. Dans M. Baker, S. Järvelä, et J. Andriessen, (dir.), Affective Learning Together (p. 251-271). Routledge.

Baker, M., Andriessen, J., Lund, K. (2009). Socio-relational, affective and cognitive dimensions of CSCL interactions: integrating theoretical methodological perspectives. Dans C. O'Malley, D. Suthers, P. Reimann et A. Dimitracopoulou (dir.), Computer Supported Collaborative Learning Practices (Vol. II, p. 31-33). International Society of the Learning Sciences.

Baker, M., Järvelä, S., et Andriessen, J. (dir.) (2013). Affective Learning Together:Social and Emotional Dimensions of Collaborative Learning. Routledge.

Beaupère, N., et Boudesseul, G. (dir.) (2009). Sortir sans diplôme de l'Université. Comprendre les parcours d'étudiants « décrocheurs ». La Documentation Française.

Beauvais, M. (2004). Des principes éthiques pour une philosophie de l'accompagnement. Savoirs, $3(6), 99-113$.

Beauvais, M. et Haudiquet, A. (2012). La recherche-accompagnement : des postures en retrait pour des visées autonomisantes. Pensée plurielle, 30-31(2), 165-174.

Bressoux, P. et Lima L. (2003). Evaluation d'une expérimentation CP à effectifs réduits [Rapport pour le Ministère de l'Education Nationale]. Direction de l'Evaluation et de la Prospective. 
Bressoux, P., et Pansu, P. (2003). Quand les enseignants jugent leurs élèves. Presses Universitaires de France.

Bressoux, P., Gurgand, M., Guyon, N., Monnet, M. et Pernaudet, J. (2016, 13 mars). Evaluation des programmes de réussite éducative [Rapport pour l'Institut des Politiques Publiques].

Conseil national d'évaluation du système scolaire. (2016). Inégalités sociales et migratoires. Comment l'école amplifie-t-elle les inégalités? CNESCO.

Delcambre, I. et Lahanier-Reuter, D. (2010). Les littéracies universitaires : Influence des disciplines et du niveau d'étude dans les pratiques de l'écrit. Dans C. Blaser et M.-C. Pollet (dir.), L'appropriation des écrits universitaires (p. 11-43). Presses Universitaires de Namur.

Desombre, C., Delelis, G., Lachal, M., Urban, E., Roye, L., Gaillet, F. et Antoine, L. (2008).

Stéréotypes de la difficulté scolaire : un outil de recueil. L'orientation scolaire et professionnelle, 37/2. https://journals.openedition.org/osp/1673

Doise, W., et Mugny, G. (1981). Le développement social de l'intelligence. InterEditions.

Duru-Bellat, M. (2002). Les inégalités sociales à l'école. PUF.

Ehrenberg, A. (2000). La fatigue d'être soi, Dépression et société. Éditions Odile Jacob.

Félix, C. et Saujat, F. (2008). L'aide au travail personnel des élèves entre déficit de prescriptions et « savoirs méthodologiques » : un double regard didactique et ergonomique. Les dossiers des sciences de l'éducation, 20, 123-136.

Félix, C. Saujat, F. et Combes, C. (2012). Des élèves en difficulté aux dispositifs d'aide : une nouvelle organisation du travail enseignant? Recherches en Education, hors-série 4, 19-30.

Feyfant, A. (2016). La différenciation pédagogique en classe. Dossier de veille de l'IFÉ, 113.

Gagnon, M. (2005). Guide pratique pour l'animation d'une communauté de recherche philosophique. Les Presses de l'Université Laval.

Good, T. L., Brophy, J. E. (2000). Looking in classrooms (8 $8^{\mathrm{e}}$ édition). Longman.

Hamilton, M. et Barton D. (2001). Editorial. Special Issue: Literacy, Home and Community. Journal of Research in Reading, 24(3), 217-221.

Holec, H. (1991). Autonomie de l'apprenant : de l'enseignement à l'apprentissage. Education Permanente, (107). http://epc.univ-lorraine.fr/EPCT_F/pdf/Autonomie.pdf

Jussim, L. et Harber, K. D. (2005). Teacher expectations and self-fulfilling prophecies: knowns and unknowns, resolved and unresolved controversies. Personality and social psychology review, 9, 131-155.

Lescouarch, L. (2011). Spécificité des pratiques pédagogiques des maîtres E en RASED. Editions universitaires européennes.

Lipman, M. (2003). Thinking in education. Cambridge University Press.

Little, D. (2010). Learner autonomy, inner speech and the European Language Portfolio. Dans Actes de la $14^{e}$ conférence internationale de l'Association Grecque de Linguistique Appliquée, Advances in Research on Language Acquisition and Teaching - Selected papers. GALA.

Madon, S., Jussim, L. et Eccles, J. (1997). In search of the powerful self-fulfilling prophecy. Journal of personality and social psychology, 72, 791-809.

Mercer, N. (1996). The quality of talk in children's collaborative activity in the classroom. Learning and instruction, 6(4), 359-377. 
Mingat, A. (1991). Les activités de rééducation GAPP à l'école primaire. Revue Française de Sociologie, 32, 515-549.

Paul, M. (2004). L'accompagnement : une posture professionnelle spécifique. L'Harmattan.

Paul, M. (2012). L'accompagnement comme posture professionnelle spécifique - L'exemple de l'éducation thérapeutique du patient, ARSI 3, (110), 13-20.

Paul, M. (2014). Passage à l'écriture et écriture du passage. Accompagner le changement au travers d'un dispositif d'écriture. Les dossiers des sciences de l'éducation, 31.

Perret, C. (2007), Quitter sa région pour entrer à l'université : quels sont les facteurs explicatifs de la mobilité géographique des bacheliers? L'orientation scolaire et professionnelle], 36(3). https:// journals.openedition.org/osp/1441

Polo, C., Lund, K., Plantin, C. et Niccolai, G. P. (2016). Group Emotions : The Social and Cognitive Functions of Emotions in Argumentation. International Journal of Computer-Supported Collaborative Learning, 11(2), 123-156.

Potvin, P. et Rousseau, R. (1993). Attitudes des enseignants envers les élèves en difficulté scolaire. Revue canadienne de l'éducation, 18, 132-144.

Proulx, J. (2004). Apprentissage par projet. Presses de l'Université du Québec.

Reverdy, C. (2017). L'accompagnement à l'école : dispositifs et réussite des élèves. Dossier de veille de l'IFÉ, 119.

Rispail, M. (2011). Littéracie: une notion entre didactique et socio-linguistique. Forum lecture. https://www.forumlecture.ch/fokusartikel1_2011_1.cfm.

Roiné, C. (2014). L'élève en difficulté : retours sur une psychologisation du social. La nouvelle revue de l'adaptation et de la scolarisation, 2(66), 13-30.

Rojas-Drummond S., Maine F., Alarcón M., Trigo A. L., Barrera M. J., Mazón N., Vélez M., Hofmann, R. (2017). Dialogic literacy : Talking, reading and writing among primary school children. Learning, Culture and Social Interaction, 12, 45-62.

Rosenthal, R. et Jacobson, L. (1968). Pygmalion in the classroom. Teacher expectations and pupils intellectual development. Holt, Rinehart and Winston.

Scott, P. H., Mortimer, E. F. et Aguiar, O. G. (2006). The tension between authoritative and dialogic discourse:A fundamental characteristic of meaning making interactions in high school science lessons. Science Education, 90, 605-663.

Soulié, C. (1995). Apprentis philosophes et apprentis sociologues. Sociétés contemporaines, 21, Les mondes des jeunes, 89-101.

Suchaut, B. (2008). Hétérogénéité des apprentissages et efficacité pédagogique. Dans Séminaire interacadémique "Enseigner en réseaux "ambition réussite" : le diagnostic scolaire au service de l'action pédagogique" ", Direction générale de l'Enseignement scolaire, Ministère de l'Education nationale, Paris, France.

Tessier, O. et Schmidt, S. (2007). Élèves à risque : origine, nature du concept et son utilisation en contexte scolaire. Revue des sciences de l'éducation, 33(3), 559-578.

Todorov, T. (1981). Mikhail Bakhtine : le principe dialogique. Le Seuil.

Von Aufschnaiter, C., Erduran, S., Osborne, J., et Simon, S. (2008). Arguing to learn and learning to argue: Case studies of how students' argumentation relates to their scientific knowledge. Journal of Research in Science Teaching, 45(1), 101-131. 


\section{NOTES}

1. Loi française $n^{\circ} 2018-166$ du 8 mars 2018 relative à l'Orientation et à la Réussite des Etudiants.

2. Cet évènement public peut prendre des formes variées, pourvu qu'il engage à la fois la présentation d'un apport sérieux sur la thématique travaillée, et une interaction avec un auditoire. Le semestre de lancement du dispositif, chacun des 4 groupes CLEFS a préparé respectivement les 4 évènements suivants : une exposition "Voyages en espaces publics ", dont l'inauguration a permis l'ouverture d'un dialogue avec d'autres étudiants et étudiantes de la filière concernée, un débat "minuté » avec plusieurs candidats et candidates aux élections européennes sur leurs propositions concernant la jeunesse, une mise en scène argumentative sous forme de disputatio sur la nature de l'Etat, une conférence théâtralisée «Violence des images, images de la violence ».

3. Le terme "élèves" n'est pas réservé, dans mon propos, aux enseignements primaire et secondaire, et s'applique parfois, comme ici, au public universitaire. Ce choix d'un terme épicène est à visée inclusive et permet de limiter, dans cet article, la formule longue « étudiants et étudiantes ».

4. La première année de licence est organisée à l'université Lyon 2 sous forme de "portails» pluridisciplinaires, associant différents départements, pour permettre aux étudiants et étudiantes de découvrir plusieurs disciplines avant de choisir une spécialisation en L2. Ainsi, par exemple, si l'on souhaite étudier les sciences de l'information et de la communication, on est inscrit en L1 dans le portail « Médias, Cultures, Sociétés ", où l'on découvre cette discipline, mais également l'anthropologie, la sociologie et les arts du spectacle. En L2, il est alors possible de poursuivre, sans procédure de réorientation, dans n'importe laquelle des 4 disciplines étudiées en L1.

5. En effet, dans les portails concernés, personne n'a été admis avec la mention " oui si ». Par conséquent, l'inscription dans le dispositif n'a pas été réalisée automatiquement mais à partir du repérage de difficultés par l'équipe enseignante (tout particulièrement les chargés de TD de Méthodologie), sur la base du volontariat (les étudiants étant libres d'accepter ou non la proposition d'intégrer le parcours).

6. Duru-Bellat a en effet montré qu'augmenter le nombre d'heures d'enseignement pouvait avoir un effet négatif, du fait d'un « rendement décroissant du temps alloué aux études au-delà du temps habituel » (2002, p. 129).

7. Issue des cercles ouvriers et pratiquée dans l'éducation populaire, l'arpentage renvoie à un ensemble de méthodes de lecture et d'appropriation collective de textes de nature variée. En annexe 2 est reproduite la fiche pédagogique présentant les 4 heures dédiées à l'arpentage dans le dispositif CLEFS.

\section{ABSTRACTS}

The CLEFS program, which has been implemented in November 2018, in Lyon, supports students with special needs in their first year at university, to prevent them from dropping out. Dramatical means invested in this program make it possible to provide students with intense support: groups of maximum 12 people, tutoring, tripled time for transversal teaching on university methodology and literacy, critical and scientific thinking. A collaborative projectbased approach enables us to address such skills in a real life perspective, aiming at organizing a 
final public event on the interdisciplinary issue studied. In this context, we intend to support their academic literacy through pedagogical tools fostering students' autonomy, with a special focus on making sense of the use or production of written materials. Dialogue plays a great role in this meaning making process, since learning and reasoning may only occur in interaction with peers, with the didactical objects studied, with existing literature, and with the audience to whom the project is directed.

Le dispositif Créer Lire Ecrire et Faire de la Science pour l'Université, expérimenté depuis novembre 2018, à Lyon 2, accompagne des étudiants et étudiantes de première année de licence1, pour prévenir leur décrochage. D'importants moyens permettent des conditions d'enseignement rares à l'université : 12 personnes maximum par groupe de travaux dirigés, tutorat, triplement du volume horaire dédié aux méthodologie et littéracie universitaires et au développement de l'esprit critique et scientifique. A travers un travail collaboratif en mode projet portant sur une thématique interdisciplinaire, ces compétences sont rendues nécessaires à l'objectif final d'organisation d'un évènement public. Ce contexte est propice à un accompagnement à la littéracie universitaire qui rende les élèves acteurs de leurs apprentissages, en insistant sur le sens du recours à l'écrit. Ce sens réside principalement dans la nécessité de dialoguer pour apprendre et raisonner; dialogue avec les pairs, avec un objet d'étude, avec la littérature, avec le public à qui on destine son travail.

\section{INDEX}

Mots-clés: accompagnement, enseignement dialogique, littéracie, mode projet, travail collaboratif

\section{AUTHOR}

\section{CLAIRE POLO}

ISPEF, 86, rue Pasteur, 69007 Lyon, claire1.polo@univ-lyon2.fr 